Selling When Brand Image Matters

Stefan Bühler and Daniel Halbheer

May 2010 Discussion Paper no. 2010-14 


$\begin{array}{ll}\text { Editor: } & \text { Martina Flockerzi } \\ & \text { University of St. Gallen } \\ & \text { Department of Economics } \\ & \text { Varnbüelstrasse 19 } \\ & \text { CH-9000 St. Gallen } \\ & \text { Phone } \quad+41712242325 \\ & \text { Fax } \quad+41712243135 \\ & \text { Email vwaabtass@unisg.ch } \\ & \text { Department of Economics } \\ & \text { University of St. Gallen } \\ & \text { Varnbüelstrasse 19 } \\ \text { Publisher: } & \text { CH-9000 St. Gallen } \\ & \text { Phone +41 71 224 23 25 } \\ & \text { Fax } \quad+41712243135 \\ & \text { http://www.vwa.unisg.ch }\end{array}$




\section{Selling When Brand Image Matters ${ }^{1}$}

Stefan Bühler and Daniel Halbheer

Author's address:

Prof. Dr. Stefan Bühler

Institute of Public Finance and Fiscal Law (IFF-HSG)

Varnbüelstrasse 19

$\mathrm{CH}-9000$ St.Gallen

Email_stefan.buehler@unisg.ch

Website www.iff.unisg.ch

Dr. Daniel Halbheer

University of Zurich

Institute for Strategy and Business Economics (ISU)

Plattenstrasse 14

$\mathrm{CH}-8032$ Zurich

Email_daniel.halbheer@isu.uzh.ch

\footnotetext{
${ }^{1}$ We thank Simon Anderson, Ernst Fehr, Martin Kolmar, and Christian Th“ oni, as well as seminar participants at the University of St. Gallen, the University of Virginia, the University of Zurich, the GEABA 2009 (Vallendar), and at the Workshop on Industrial Organization: Theory, Empirics and Experiments (Lecce, 2009) for most helpful comments and suggestions. Financial support from the Swiss National Science Foundation through grants PP0012-114754 (Buehler) and PA00P1-129097 (Halbheer) is gratefully acknowledged. Daniel Halbheer thanks the Department of Economics at the University of Virginia for being a marvelous host while some of this research was being undertaken.
} 


\begin{abstract}
This paper studies profit-maximizing seller behavior when brand image affects consumer demand. We consider a seller facing a population of consumers with heterogeneous tastes regarding product quality and brand image. First, we analyze "active branding" by the seller through costly advertising. Our analysis shows that advertising, price and profits are all increasing in the average valuation of brand image in the population. Second, we examine the role of "passive branding" emanating from the population's consumption of the product. We demonstrate that seller profits increase in the average degree of conformity in the opulation whereas the price remains unaffected.
\end{abstract}

\title{
Keywords
}

Quality; brand image; advertising; conformity; exclusivity.

\section{JEL Classification}

D42, L15, L21, M37. 


\section{Introduction}

Selling products means catering to the tastes of consumers. From a seller's point of view, catering to consumer tastes is complicated by the fact that tastes are heterogeneous and not necessarily restricted to the tangible characteristics of a given product. It is well known, for instance, that a consumer's decision whether to purchase a product may be influenced by his perception of the brand image associated with the product.

Consumers are especially susceptible to brand image when consumption is public and subject to social assessment. Consider, for example, the decision to purchase a new car. In addition to evaluating its tangible characteristics (horsepower, fuel efficiency, etc.), a consumer is likely to account for the brand image that is associated with the car (cf. Meenaghan 1995). Similarly, a consumer's decision whether to buy an MP3 player is likely do depend on the tangible characteristics (e.g., memory, display size) as well as the brand image of the player. In light of the important role that brand image plays in many markets, it is surprising that, to the best of our knowledge, there is no formal analysis of profit-maximizing seller behavior when brand image matters for consumers.

The purpose of this paper is to study profit-maximizing seller behavior when consumer demand is affected by brand image. We analyze the behavior of a seller facing a population of consumers with heterogeneous tastes regarding tangible product quality and brand image. Consumers either derive brand image from the seller's costly advertising ("active branding") or the population's consumption of the product ("passive branding"). ${ }^{1}$ Our analysis assumes that consumer tastes regarding product quality and brand image are distributed according to a bivariate uniform distribution which is common knowledge. Individual tastes, however, are private information. Importantly, this specification implies that different consumers have different valuations of product quality and brand image.

In a first step, we characterize consumer demand as a function of product price and brand image. We show that, depending on the parameters of the type distribution, four different cases need to be distinguished. The differences between these cases relate to the importance that the perceived brand image may have relative to the valuation of product quality. Our analysis focuses on the case where the perception of brand image does not "dominate" the valuation of product quality. ${ }^{2}$

\footnotetext{
${ }^{1}$ The literature on branding commonly assumes that brand image is built on the product itself. See, for instance, Keller and Lehmann (2006).

${ }^{2}$ This case is closest to the standard monopoly model of vertical differentiation (see below).
} 
In a second step, we employ our demand framework to study optimal seller behavior. We first investigate active branding by the seller through costly advertising. To do so, we impose some more structure on the model. For simplicity, we adopt a reduced-form approach and assume that the firm's advertising intensity determines brand image. ${ }^{3}$ We also assume that the average consumer's valuation of brand image is positive (even though some consumers may dislike it) to make active branding profitable. For this setting, we find that advertising, price and profits are all increasing in the average consumer's valuation of brand image.

Next, we examine the role of passive branding emanating from the population's consumption of the product. In this alternative setting, brand image stems from consumption externalities rather than costly advertising. We assume that brand image is determined by the number of consumers who purchase the product and impose rational expectations on behalf of consumers without explicitly modeling the process of forming expectations (Katz and Shapiro 1985). A consumer has a concern for conformity (exclusivity, respectively) if he is better off the higher (lower) the number buyers (see, for instance, Amaldoss and Jain 2005a, b). We find that demand and seller profits are increasing in the average degree of conformity, whereas pricing remains unaffected.

This paper is related to the advertising literature surveyed in Bagwell (2007). First, our analysis suggests that, on average, active branding works similarly to persuasive advertising which affects consumer preferences by increasing the willingness to pay (cf. von der Fehr and Stevik 1998). The key difference is that, in our framework, active branding may actually decrease the willingness to pay for a subset of consumers (those with a distaste for the brand image). Second, our analysis of passive branding is related to the literature on conspicuous consumption. Amaldoss and Jain (2005a) also employ a monopoly model and examine how purchase decisions are affected by the desire for exclusivity and conformity, respectively. Yet, these authors do not allow for heterogeneous tastes regarding quality. Bernheim (1994), Pesendorfer (1995), Bagwell and Bernheim (1996), Corneo and Jeanne (1997), and Krähmer (2006) provide signaling models of conspicuous consumption. Our approach is different in that we model conformity and exclusivity using consumption externalities. Third, our paper adds to the literature on vertical differentiation (see, e.g., Mussa and Rosen 1978, Gabszewicz and Thisse 1979, Shaked and Sutton 1982, Baake and Boom 2001). The standard monopoly model of vertical differentation is a special case of our model in which brand image does not matter.

\footnotetext{
${ }^{3}$ For instance, Krähmer (2006) shows that (persuasive) advertising creates a brand's image employing a signaling model.
} 
The paper proceeds as follows. Section 2 details the analytical framework and introduces consumer preferences that account for brand image. Section 3 derives consumer demand as a function of the product price and brand image. Using this demand framework, Section 4 studies, respectively, active branding and passive branding as mechanisms for building brand image and characterizes optimal firm behavior. Section 5 discusses conclusions and directions for future research.

\section{Model}

We model the provision of a branded product by a profit-maximizing seller. The production of the good may involve a fixed cost $F \geq 0$, and the marginal costs of output are assumed to be constant and normalized to zero. ${ }^{4}$ The seller faces a population of potential consumers with mass $N$, where $N$ is normalized to unity without loss of generality, and offers its product at price $p$.

The consumers' complete experience of the product depends on both intrinsic product quality and "brand intangibles" (Keller and Lehmann, 2006). Intrinsic product quality is described by the real-valued index $v$ that summarizes the various aspects of quality (Lancaster, 1966). Brand intangibles $b$ in contrast reflect image attributes such as either accompanying advertising ("active branding") or the consumption of the product by others ("passive branding"). We assume that consumers' valuations $\theta$ of product quality $v$ are uniformly distributed on the interval $[0,1]$. We also assume that consumers differ in their perception of brand image $b$, captured by a taste parameter $\sigma$ that follows a uniform distribution on $[\underline{\sigma}, \bar{\sigma}]$, with $\underline{\sigma}<0$ and $\bar{\sigma}>0$. The expectation of $\sigma$ is denoted by $\mu_{\sigma} \equiv(\underline{\sigma}+\bar{\sigma}) / 2$.

Specifically, we let a consumer's conditional indirect utility from buying the product be given by

$$
V(p, y ; v, b, \theta, \sigma)=\theta v+\sigma b+y-p,
$$

where $y$ denotes income and $y-p$ has the interpretation of utility derived from consuming an outside (composite) good. Importantly, the utility difference between consuming the good and not consuming the good, $V(p, y ; v, b, \theta, \sigma)-y$, depends on brand image. That is, consumers derive more utility from consuming the good when they value the product's brand image $(\sigma>0)$ and less when they dislike the brand image $(\sigma<0)$. The taste parameters $\theta$ and $\sigma$ are independently drawn from their respective underlying distributions. This assumption

\footnotetext{
${ }^{4}$ We assume that the fixed cost do not exceed the firm's profit. Hence, they do not change the analysis and can therefore be omitted.
} 
means that the valuations of quality and brand image are uncorrelated. ${ }^{5}$ We further assume that the distributions of $\theta$ and $\sigma$ are common knowledge. The realization of type $t \equiv(\theta, \sigma)$, however, is private information.

\section{Consumer Demand}

This section derives consumer demand for the product as a function of the price $p$ and brand image $b$. Our assumptions on the distributions of $\theta$ and $\sigma$, respectively, imply that consumer types $t$ are distributed according to the bivariate uniform distribution

$$
f_{(\Theta, \Sigma)}(\theta, \sigma)= \begin{cases}(\bar{\sigma}-\underline{\sigma})^{-1} & \text { if }(\theta, \sigma) \in T \\ 0 & \text { otherwise }\end{cases}
$$

on the rectangular type space $T \equiv[0,1] \times[\underline{\sigma}, \bar{\sigma}]$.

Clearly, a consumer does not purchase the product if he attains a lower utility than when choosing the outside good. Put differently, a consumer does not purchase if the consumption utility, $\theta v+\sigma b$, is less than (or equal to) the price $p$. Letting $F(p \mid b)$ denote the distribution function of $\theta v+\sigma b$ for given $b$, the mass (or fraction) of consumers who do not purchase can be expressed as

$$
\begin{aligned}
F(p \mid b) & \equiv \operatorname{Pr}\{\theta v+\sigma b \leq p\}=\iint_{\{\theta v+\sigma b \leq p\}} f_{(\Theta, \Sigma)}(\theta, \sigma) d \theta d \sigma \\
& =\frac{1}{(\bar{\sigma}-\underline{\sigma})} \int_{\underline{\sigma}} \int_{0}^{\bar{\sigma}} \mathbf{1}_{\{\theta v+\sigma b \leq p\}} d \theta d \sigma,
\end{aligned}
$$

where $\mathbf{1}_{A}$ is the indicator function of the set $A$. The demand for the product is thus given by

$$
D(p, b) \equiv 1-F(p \mid b)=1-\frac{1}{(\bar{\sigma}-\underline{\sigma})} \int_{\underline{\sigma}}^{\bar{\sigma}} \int_{0}^{1} \mathbf{1}_{\{\theta v+\sigma b \leq p\}} d \theta d \sigma .
$$

Note that, by the definition of a distribution function, there is a monotone relationship between the price $p$ and the probability of buying, $\operatorname{Pr}\{\theta v+\sigma b \geq p\}$, which in turn implies a downward-sloping demand curve.

Consumer demand as well as the set of buying consumers,

$$
B \equiv\{(\theta, \sigma) \mid \theta v+\sigma b \geq p\},
$$

\footnotetext{
${ }^{5}$ Alternatively, one could argue either that those with high valuations of quality tend to highly value brand image (positive correlation) or that those with the highest perceptions of brand image are least concerned about product quality (negative correlation). Similarly, those with low valuations of quality may care less about brand image or highly value brand image.
} 


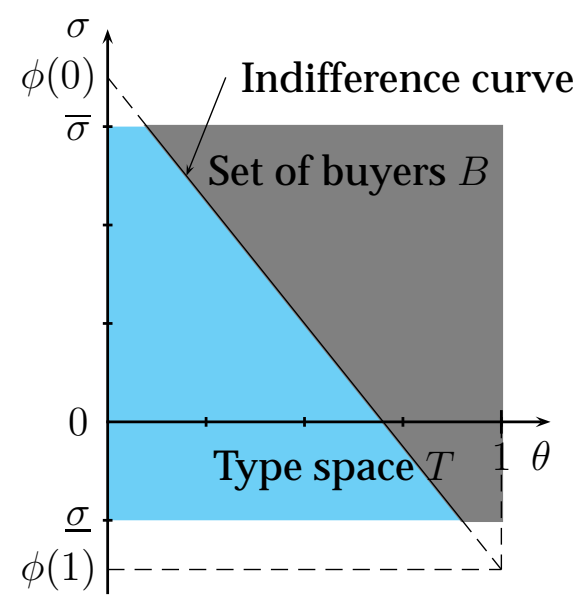

(A) Case 1

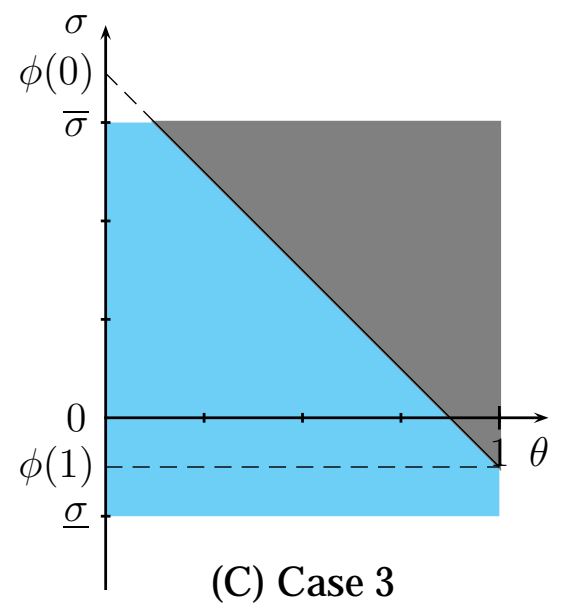

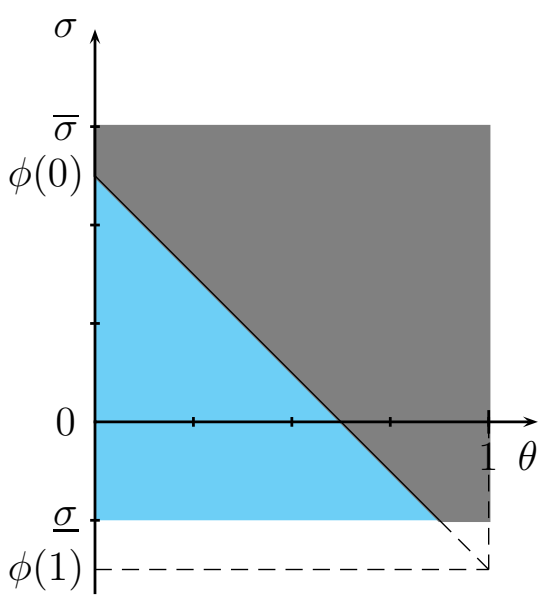

(B) Case 2

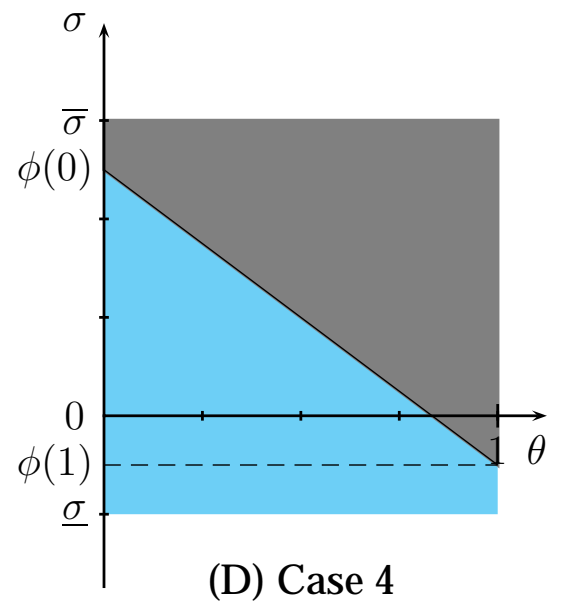

Figure 1: Four type distributions.

crucially depends on the distribution of brand perceptions $\sigma$. Clearly, for extremely low prices $(p \leq \underline{\sigma} b)$ all consumers purchase $(D(p, b)=1)$, whereas for extremely high prices $(p \geq v+\bar{\sigma} b)$, no consumer purchases $(D(p, b)=0)$. For intermediate prices $p \in(\underline{\sigma} b, v+\bar{\sigma} b)$, a consumer is indifferent between buying and not buying if his type $t$ satisfies the "indifference condition"

$$
\sigma=\frac{p-\theta v}{b} \equiv \phi(\theta) .
$$

Depending on the parameters of the distribution of brand perceptions, $\underline{\sigma}$ and $\bar{\sigma}$, four different cases emerge (see Figure 1 and Table 1, respectively).

If $\phi(0)>\bar{\sigma}$ and $\phi(1) \leq \underline{\sigma}$ (Case 1), for instance, none of the consumers with 


\begin{tabular}{lcc}
\hline & $\begin{array}{c}\text { Low-WTP Consumers } \\
(\theta=0)\end{array}$ & $\begin{array}{c}\text { High-WTP Consumers } \\
(\theta=1)\end{array}$ \\
\hline Case $\mathbf{1}$ & None Buy & All Buy \\
$\phi(0)>\bar{\sigma}$ and $\phi(1) \leq \underline{\sigma}$ & $p>\bar{\sigma} b$ & $p \leq v+\underline{\sigma} b$ \\
Case $\mathbf{2}$ & Some Buy & All Buy \\
$\phi(0) \leq \bar{\sigma}$ and $\phi(1) \leq \underline{\sigma}$ & $p \leq \bar{\sigma} b$ & $p \leq v+\underline{\sigma} b$ \\
Case $\mathbf{3}$ & None Buy & Some Buy \\
$\phi(0)>\bar{\sigma}$ and $\phi(1)>\underline{\sigma}$ & $p>\bar{\sigma} b$ & $p>v+\underline{\sigma} b$ \\
Case $\mathbf{4}$ & Some Buy & Some Buy \\
$\phi(0) \leq \bar{\sigma}$ and $\phi(1)>\underline{\sigma}$ & $p \leq \bar{\sigma} b$ & $p>v+\underline{\sigma} b$ \\
\hline
\end{tabular}

Table 1: Willingness to pay (WTP) and demands for prices $p \in(\underline{\sigma} b, v+\bar{\sigma} b)$.

the lowest willingness to pay (WTP) for quality $(\theta=0)$ purchases the product, whereas all consumers with the highest willingness to pay $(\theta=1)$ buy. Panel A in Figure 1 illustrates the type space $T$ along with the indifference curve and the corresponding set of buyers $B$. If instead $\phi(0) \leq \bar{\sigma}$ and $\phi(1) \leq \underline{\sigma}$ (Case 2), some consumers with the lowest willingness to pay for quality purchase the product (those with the highest $\sigma^{\prime}$ s), and all consumers with the highest willingness to pay buy (Panel B in Figure 1). The other two cases emerge if some consumers with the highest willingness to pay for quality buy $(p>v+\underline{\sigma} b)$, and either none ( $p>\bar{\sigma} b$, Case 3 ) or some $(p \leq \bar{\sigma} b$, Case 4$)$ of the consumers with the lowest willingness to pay purchase the product (Panels $C$ and D, respectively, in Figure 1).

Our analysis of active and passive branding below will be based on the demand function emerging in Case 1. This choice is motivated by our view that it is natural to start from a setting where the valuation of intrinsic product quality is not "dominated" by the perception of brand image. That is, we effectively impose that (i) no consumer buys solely because of the brand image, and (ii) a distaste for the brand image does not discourage consumers with the highest valuation of quality from buying. Clearly, brand image nevertheless affects the purchase decision of many consumers in the population (see Figure 1). Lemma 1 gives the relevant demand function.

Lemma 1. For prices $p \in(\bar{\sigma} b, v+\underline{\sigma} b]$, consumer demand is given by

$$
D(p, b)=1-\frac{p}{v}+\frac{b \mu_{\sigma}}{v} .
$$


Proof. See the Appendix. ${ }^{6}$

Three remarks on Lemma 1 are in order. First, consumer demand depends on brand image only through $\mu_{\sigma}$ (and $v$ ). That is, from the firm's point of view, all that matters is the average consumer's valuation of brand image. This immediately implies that brand image is not relevant for profit-maximizing seller behavior if the distribution of consumer tastes regarding brand image is symmetric around zero. Second, if the average consumer positively values brand image $\left(\mu_{\sigma}>0\right)$ branding has a demand-enhancing effect. Conversely, branding has a demand-reducing effect if the average consumer dislikes the brand image $\left(\mu_{\sigma}<0\right)$. Third, observe that a higher intrinsic product quality $v$ increases demand, whereas a higher price $p$ leads to lower demand.

\section{Seller Behavior}

In this section we study two mechanisms for building brand image. Depending on the notion of brand image, the firm may either advertise to stimulate the demand for its product ("active branding") or price its product by taking into account that consumers care about the use of the product by other consumers ("passive branding"). The benchmark model to which we shall sometimes compare our results is the monopoly model of vertical (quality) differentiation without brand image.

\subsection{Active Branding}

We first consider active branding by the firm through costly advertising. We describe the setup of the model and then characterize profit-maximizing seller behavior. Our main focus will be on investigating how tastes regarding brand image affect seller behavior.

\subsubsection{Setup}

Suppose that the seller chooses both the price $p$ and the brand image $b$ he likes to cultivate in the market place. Although brand image is typically determined by multiple marketing activities, we employ a reduced-form approach and suppose that it is the advertising intensity captured by the index $b>0$ which determines brand image. We assume that the investment in brand image $b$ involves quadratic advertising outlays $\gamma b^{2}$, where $\gamma \in \mathbb{R}_{+}$is large enough for the cost function to be sufficiently convex (see below). In this context, the taste

\footnotetext{
${ }^{6}$ All proofs can be found in the Appendix. The appendix also provides the demand functions for the other cases and shows how they are related.
} 
parameter $\sigma$ may be interpreted as measure for ad-sensitivity. Consumers are ad-lovers if $\sigma>0$ and ad-avoiders if $\sigma<0 .{ }^{7}$ We assume that $\mu_{\sigma}>0$, that is, we restrict attention to the case where advertising has a demand-enhancing effect (which is equivalent to assuming that the majority of consumers are ad-lovers).

\subsubsection{Analysis}

For convenience, we view the firm's profit-maximizing problem as a two-stage decision problem and suppose that the decision about the advertising level precedes the pricing decision. ${ }^{8}$ Therefore, the optimal price for a given advertising level $b$ solves the problem

$$
\max _{p} \pi\left(p ; b, \mu_{\sigma}\right)=p\left(1-\frac{p}{v}+\frac{\mu_{\sigma} b}{v}\right)-\gamma b^{2},
$$

where firm profits are given by revenues (recall that marginal costs of output are zero) net of the cost of advertising. Solving the necessary and sufficient first-order condition we obtain

$$
p\left(b ; \mu_{\sigma}\right)=\frac{v}{2}\left(1+\frac{\mu_{\sigma} b}{v}\right) .
$$

Substituting the optimal price back into the objective function, we obtain the seller's profit as a function of brand image:

$$
\pi(b)=\frac{v}{4}\left(1+\frac{\mu_{\sigma} b}{v}\right)^{2}-\gamma b^{2}
$$

The solution to the necessary and sufficient first-order yields the optimal investment in brand image

$$
b^{*}\left(\mu_{\sigma}\right)=\frac{\mu_{\sigma} v}{4 \gamma v-\mu_{\sigma}^{2}} .
$$

Straightforward calculations then yield the seller's price, demand, and profits in terms of model parameters (highlighting the dependence on $\mu_{\sigma}$ ) $:^{9}$

$$
p^{*}\left(\mu_{\sigma}\right)=\frac{2 \gamma v^{2}}{4 \gamma v-\mu_{\sigma}^{2}}, \quad D^{*}\left(\mu_{\sigma}\right)=\frac{2 \gamma v}{4 \gamma v-\mu_{\sigma}^{2}}, \quad \text { and } \quad \pi^{*}\left(\mu_{\sigma}\right)=\frac{\gamma v^{2}}{4 \gamma v-\mu_{\sigma}^{2}} .
$$

Our first proposition summarizes how the seller's behavior is related to the distribution of consumer tastes regarding brand image.

\footnotetext{
${ }^{7}$ This terminology is used by various authors (see, for example, Gabszewicz et al. 2005).

${ }^{8}$ Simultaneous decisions would lead to the same results.

${ }^{9}$ We assume that $\gamma \geq\left(\mu_{\sigma}^{2}-\underline{\sigma} \mu_{\sigma}\right) / 2 v$. Under this assumption, the brand image $b^{*}$ and the price $p^{*}$ satisfy the requirements of Lemma 1. The assumption on the cost parameter $\gamma$ also ensures an interior solution for $b$ (which in turn implies that the optimal price, demand, and profits are strictly positive) and $D^{*} \leq 1$.
} 
Proposition 1 (Active Branding). Suppose that the average consumer's valuation of brand image is strictly positive $\left(\mu_{\sigma}>0\right)$. Then, (i) the product price $p^{*}$, (ii) the level of advertising $b^{*}$, and (iii) seller profits $\pi^{*}$ are increasing in $\mu_{\sigma}$.

Proposition 1 highlights that consumer tastes regarding brand image may be an important determinant of seller behavior. If the population's average valuation of brand image is positive, the profit-maximizing price, the level of advertising, and profits are all increasing in the average consumer's valuation of brand image. Intuitively, the latter result stems from demand-markup complementarities which are common in markets with seller power (see Athey and Schmutzler 2001). These complementarities imply that investing in brand image increases both price (markup) and demand, leading to higher profits.

The result also suggests that, with $\mu_{\sigma}>0$, active branding on average works similarly to persuasive advertising (cf. von der Fehr and Stevik 1998, Bagwell 2007), which increases the consumers' willingness to pay. Note, however, that in our analysis, active branding actually reduces the willingness to pay of a subset of consumers (those with a distaste for the product's brand image). Profit-maximizing seller behavior does account for this by tailoring the advertising intensity to the taste of the average consumer in the population.

At a more fundamental level, the result highlights that "consumers get what they want": A product with given quality will be accompanied by different levels of advertising if sold to populations with different average valuations of brand image. In the case where the average valuation of brand image is negative, the profit-maximizing firm does not incur the cost of advertising since it only reduces profits.

Finally, Proposition 1 sheds light on what is known as "brand equity" in the marketing literature (cf. Farquahr 1989, Erdem et al. 1999). The notion of brand equity intends to capture the extra value that a firm garners from endowing a product with a positive brand image. Our analysis suggests that a positive brand equity requires $\mu_{\sigma}>0 .{ }^{10}$

We now briefly discuss the comparative statics properties of changes in the other model parameters. We first focus on advertising costs. Increasing $\gamma$ reduces the investment in brand image, the optimal price (notice that there is an indirect effect only as the best-response function does not depend on $\gamma$ ), demand and hence the seller's profit. Shifting attention to increases in product quality, we find that increasing $v$ leads to lower investments in brand image, a higher price (because the positive direct effect dominates the negative advertising-mediated effect), lower demand (due to the higher price and the lower investment in brand image), but still higher profits.

\footnotetext{
${ }^{10}$ In our model, brand equity may formally be calculated as $\pi\left(\mu_{\sigma}\right)-\pi(0)=\left(\mu_{\sigma}^{2} v\right) /[4(4 \gamma v-$ $\left.\left.\mu_{\sigma}^{2}\right)\right]>0$, which is positive for $\mu_{\sigma}>0$.
} 


\subsection{Passive Branding}

In this section, we consider passive branding emanating from the population's consumption of the product. Again, we first discuss the setup of the model and then characterize the profit-maximizing seller behavior. The main focus of the analysis will be on investigating how tastes regarding the consumption of others affects optimal seller behavior.

\subsubsection{Setup}

Suppose that the seller's only decision variable is the price $p$ and let brand image be captured by the index $b$, which is determined by the population's consumption of the product. More specifically, we suppose that $b$ is determined by the expected number of consumers $D^{e}(p)$ who purchase the product at price $p .{ }^{11}$ That is, passive branding is driven by consumption externalities among heterogeneous individuals. In line with the economic analysis of networks, we impose rational expectations on behalf of consumers without explicitly modeling the process of forming expectations (Katz and Shapiro 1985).

In this setting, $\sigma$ reflects a consumer's taste regarding the consumption of others, that is, his "social attitude". A consumer with a taste parameter $\sigma>0$ is called a conformist, because he is better off the higher the (expected) number of consumers who purchase the product (cf. Clark and Oswald 1998, Grilo et al. 2001, Amaldoss and Jain 2005a, b). Conversely, a consumer with a taste parameter $\sigma<0$ is called an exclusivist, because he is worse off the higher the number of consumers. In the subsequent analysis, we let the expectation of $\sigma$, $\mu_{\sigma}$, represent the average degree of conformity in the population. In contrast to our above analysis of active branding, we leave the sign of $\mu_{\sigma}$ unrestricted. ${ }^{12}$ We require, however, that the average degree of conformity in the population is sufficiently small relative to the average valuation of product quality (see below). This assumption is consistent with our focus on demand where the valuation of intrinsic product quality is not dominated by the valuation of brand image.

\subsubsection{Analysis}

Under the assumption of fulfilled expectations, consumers' expected demand must be equal to actual demand. Thus, expected demand $D^{e}(p)$ must satisfy the fixed point condition $D^{e}(p)=D\left(p ; D^{e}(p)\right)$, from which we can infer the

\footnotetext{
${ }^{11}$ In principle, consumers might also be interested in the purchase decisions of particular subgroups of the population (e.g. consumers with the highest willingness to pay for quality). For simplicity, we abstract from this possibility.

${ }^{12}$ Note that $\mu_{\sigma}<0$ if the majority of consumers are exclusivists.
} 
seller's "perceived" demand curve

$$
D(p)=\frac{v-p}{v-\mu_{\sigma}}
$$

The seller's only decision variable is the product price $p$. Given perceived demand, the seller's optimal price solves the problem

$$
\max _{p} \pi\left(p ; \mu_{\sigma}\right)=p\left(\frac{v-p}{v-\mu_{\sigma}}\right),
$$

and is given by

$$
p^{*}=\frac{v}{2} .
$$

It is immediately apparent that the optimal price does not depend on the average degree of conformity in the population. This follows from the fact that the only impact $\mu_{\sigma}$ has on perceived demand is through the scaling factor $1 /\left(v-\mu_{\sigma}\right)$. Consequently, the seller's optimal price $p^{*}$ coincides with the price in the standard monopoly model of vertical differentiation.

Substituting the optimal price back into the demand function, it is straightforward to calculate consumer demand and seller profits in terms of the model parameters (highlighting the dependence on $\left.\mu_{\sigma}\right):{ }^{13}$

$$
D^{*}\left(\mu_{\sigma}\right)=\frac{v}{2\left(v-\mu_{\sigma}\right)} \text { and } \pi^{*}=\frac{v^{2}}{4\left(v-\mu_{\sigma}\right)} .
$$

The next proposition summarizes our key results.

Proposition 2 (Passive Branding). Assume that consumers form rational expectations about the consumption of others and let $\mu_{\sigma}$ denote the average degree of conformity in the population. Then, (i) the product price $p^{*}$ is independent of $\mu_{\sigma}$ and (ii) consumer demand $D^{*}$ and seller profits $\pi^{*}$ are increasing in $\mu_{\sigma}$.

Proposition 2 highlights that the average degree of conformity may be an important determinant of consumer demand and seller profits even if it leaves the profit-maximizing price unaffected. It implies, in particular, that consumer demand in a conformistic population $\left(\mu_{\sigma}>0\right)$ is higher than in a exlusivistic population $\left(\mu_{\sigma}<0\right)$ even though prices are equal. This finding complements earlier work by Grilo et al. (2001) and Amaldoss and Jain (2005a, b) who emphasize the impact of social concerns on product prices.

\footnotetext{
${ }^{13}$ We assume $\mu_{\sigma}<v-\max \{-\underline{\sigma}, \bar{\sigma}\}$ so that the requirements of Lemma 1 are satisfied. This assumption also implies that demand and profits are strictly positive and $D^{*} \leq 1$.
} 


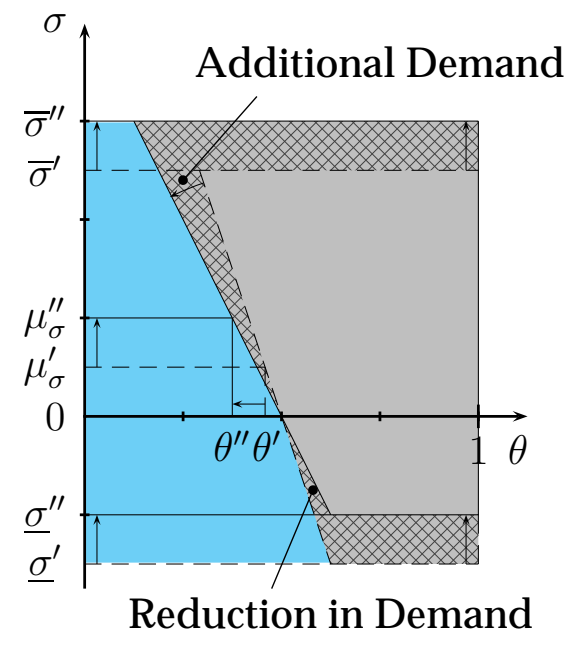

(A) Conformists

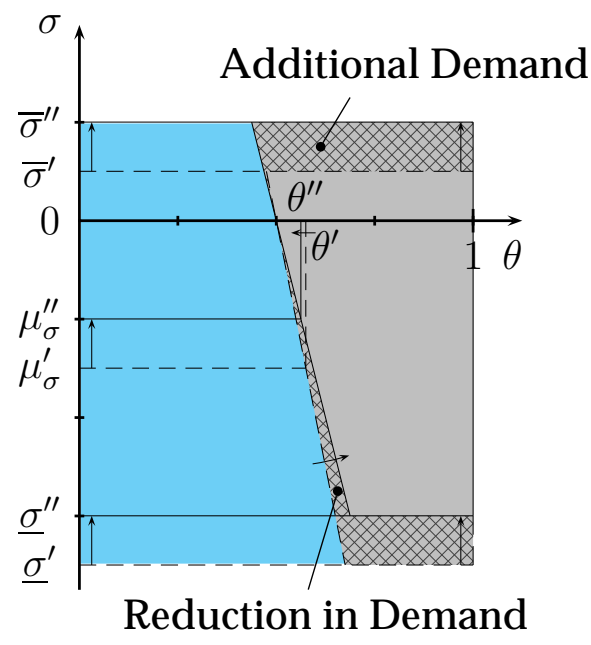

(B) Exclusivists

Figure 2: Demand effects of shifts in the type space $T$.

The result also implies that the seller of a product perceived as exclusive does not necessarily need to "restrict" the quantity it sells, for instance, by selling through exclusive distribution channels only. ${ }^{14}$ Our analysis rather suggests that, if the majority of consumers perceives the good as exclusive, consumer demand will automatically be low.

As mentioned above, result (i) of Proposition 2 follows from the fact that the average degree of conformity affects perceived demand solely through a scaling factor and therefore leaves the profit-maximizing price unaffected. The intuition for result (ii) is best understood by inspection of Figure 2, which illustrates the demand effects of an increase in the average degree of conformity $\mu_{\sigma}$ from an "upwards shift" of the type space $T$.

Let us first consider Panel A where the majority of consumers are conformists $\left(\mu_{\sigma}^{\prime}>0\right)$. Recall from Section 3 that the set of buyers $B$ is given by the area to the right of the negatively sloped straight line trough $T$ representing the indifference condition $\sigma=\phi(\theta)$. It is easy to see that the upwards shift of $T$ increases the average degree of conformity from $\mu_{\sigma}^{\prime}$ to $\mu_{\sigma}^{\prime \prime}$. In addition, it

\footnotetext{
${ }^{14}$ Another device of sellers of exclusive goods is to restrict trade by not selling over the Internet or by marketing "limited editions". See Amaldoss and Jain $(2005 a, b)$ and the references therein.
} 
adds consumers with a strong taste for conformity $\left(\sigma>\bar{\sigma}^{\prime}\right)$ to $B$ and excludes consumers with a strong taste for exclusivity $\left(\sigma<\underline{\sigma}^{\prime \prime}\right)$ from $B$. Interestingly, the upwards shift in $T$ also leads to a counter-clockwise rotation of the indifference condition $\sigma=\phi(\theta)$ around the point of intersection with the $\theta$-axis. ${ }^{15}$ That is, consumers in the neighborhood of the original indifference condition reverse their purchase decision in response to the upwards shift in $T$. Intuitively, this follows from the rational expectation of higher demand, such that conformists (exclusivists, respectively) derive a higher (lower) indirect utility from consumption. Note that the overall demand effect of the increase from $\mu_{\sigma}^{\prime}$ to $\mu_{\sigma}^{\prime \prime}$ is positive, as stated in part (ii) of Proposition 2.

Next, consider Panel B where the majority of consumers are exclusivists $\left(\mu_{\sigma}^{\prime}<0\right)$. Again, the upward shift of $T$ increases the average degree of conformity from $\mu_{\sigma}^{\prime}$ to $\mu_{\sigma}^{\prime \prime}$. This adds new consumers with $\sigma>\bar{\sigma}^{\prime}$ to $B$ and excludes consumers with $\sigma>\underline{\sigma}^{\prime \prime}$ from $B$. In addition, consumers rationally expect higher demand and revise their purchase decisions accordingly. The overall demand effect of the increase from $\mu_{\sigma}^{\prime}$ to $\mu_{\sigma}^{\prime \prime}$ is again positive.

Before concluding, we briefly discuss how changes in intrinsic product quality $v$ affect optimal quantities. Clearly, increasing $v$ calls for a higher optimal price $p^{*}$. Interestingly, demand at the optimal price $p^{*}$ is decreasing in $v$, although quality has a demand-enhancing effect. That is, the negative pricemediated effect dominates the positive direct demand effect of increasing $v$. As a result, seller profits are increasing in $v$.

\section{Conclusions}

This paper has studied profit-maximizing seller behavior when brand image affects consumer demand. Considering a monopoly seller facing consumers with heterogeneous tastes regarding product quality and brand image, we have characterized consumer demand and analyzed active branding by the seller through costly advertising and passive branding emanating from the population's consumption of the product.

We have shown that the distribution of tastes in the population is an important determinant of seller behavior: With active branding, advertising, price, and profits are all increasing in the average valuation of brand image in the population. With passive branding, seller profits are increasing in the average degree of conformity in the population, even though the price remains unaffected.

\footnotetext{
${ }^{15}$ To see this, note that an increase in $\mu_{\sigma}$ increases $D(p)$ and thus $D^{e}(p)$ (by fulfilled expectations). Since $D^{e}(p)=b$ with passive branding, the denominator in the slope $\phi^{\prime}=-v / b$ becomes larger, leading to a counter-clockwise rotation of the indifference curve.
} 
Our analysis suggests a number of avenues for future research. First, it would be interesting to study the behavior of a seller that offers several versions of the product with different brand images. Second, it would be natural to extend the analysis to a setting with imperfect competition where brand image matters. In such a setting, it is conceivable, for instance, that sellers compete in positioning their products in the quality/brand image space. Third, it would be interesting to examine settings where the sellers may be able to influence the consumers' perception of brand image (which is exogenous in our setting by assumption). Fourth, and finally, our framework for deriving demand may be generalized to other types of distributions and higherdimensional tastes. We hope to address these issues in future research.

\section{References}

Amaldoss, Wilfred and Sanjay Jain (2005a): 'Pricing of Conspicuous Goods: A Competitive Analysis of Social Effects.' Journal of Marketing Research 42(1), 30-42.

Amaldoss, Wilfred and Sanjay Jain (2005b): 'Consumption and Sophisticated Thinking.' Management Science 51(10), 1449-1466.

Athey, Susan and Armin Schmutzler (2001): 'Investment and market dominance.' RAND Journal of Economics 32(1), 1-26.

Baake, Pio and Anette Boom (2001): 'Vertical Product Differentiation, Network Externalities, and Compatibility Decisions.' International Journal of Industrial Organization 19(1-2), 267-284.

Bagwell, Kyle (2007): 'The Economic Analysis of Advertising.' In: M. Armstrong and R. Porter (eds.), Handbook of Industrial Organization, Vol. III, Elsevier Science, pp. 1701-1844.

Bagwell, Laurie S. and Bernheim, B. Douglas (1996): 'Veblen Effects in a Theory of Conspicuous Consumption.' American Economic Review 86(3), 349-373.

Bernheim, B. Douglas (1994): 'A Theory of Conformity.' Journal of Political Economy 102(5), 841-877.

Clark, Andrew E. and Andrew J. Oswald (1998): 'Comparison-Concave Utility and Following Behaviour in Social and Economic Settings.' Journal of Public Economics 70(1), 133-155.

Corneo, Giacomo and Jeanne, Olivier (1997): ‘Conspicuous Consumption, Snobbism and Conformism.' Journal of Public Economics 66(1), 55-71. 
Erdem, Tülin, Joffre Swait, Susan Broniarczyk, Dipankar Chakravarti, JeanNoel Kapferer, Michael Keane, John Roberts, Jan-Benedict Steenkamp and Florian Zettelmeyer (1999): 'Brand Equity, Consumer Learning and Choice.' Marketing Letters 10(3), 301-318.

Farquhar Peter H. (1989): 'Managing Brand Equity.' Marketing Research 1(September), 24-33.

Gabszewicz, J. Jaskold and J.-F. Thisse (1979): 'Price Competition, Quality and Income Disparities.' Journal of Economic Theory 20(3), 340-359.

Gabszwewicz, Jean J., Didier Laussel and Nathalie Sonnac (2005): 'Does Advertising Lower the Price of Newspapers to Consumers? A Theoretical Appraisal.' Economics Letters 87(1), 127-134.

Grilo, Isabel, Oz Shy, and Jacques-François Thisse (2001): 'Price Competition when Consumer Behavior is Characterized by Conformity or Vanity.' Journal of Public Economics 80(3), 385-408.

Katz, Michael L. and Carl Shapiro (1985): 'Network Externalities, Competition, and Compatibility.' American Economic Review 75(3), 424-440.

Keller, Kevin L. and Donald R. Lehmann (2006): 'Brands and Branding: Research Findings and Future Priorities.' Marketing Science 25(6), 740-759.

Krähmer, Daniel (2006): 'Advertising and Conspicuous Consumption.' Journal of Institutional and Theoretical Economics 162(4), 661-682.

Lancaster, Kelvin J. (1966): 'A New Approach to Consumer Theory.' Journal of Political Economy 74(2), 132-157.

Meenaghan, Tony (1995): 'The Role of Advertising in Brand Image Development.' Journal of Product \& Brand Management 4(4), 23-34.

Mussa, Michael and Sherwin Rosen (1978): 'Monopoly and product quality.' Journal of Economic Theory 18(2), 301-317.

Pesendorfer, Wolfgang (1995): 'Design Innovation and Fashion Cycles.' American Economic Review 85(4), 771-792.

Shaked, Avner and John Sutton (1983): 'Natural oligopolies.' Econometrica 51(5), 1469-1483.

von der Fehr, Nils-Henrik M. and Kristin Stevik (1998): 'Persuasive Advertising and Product Differentiation.' Southern Economic Journal 65(1), 113-126. 


\section{Appendix}

This appendix gives the proofs of our results.

\section{A.1 Consumer Demand}

This section derives the demand function for each of the four cases for all prices $p \in$ $(\underline{\sigma} b, v+\bar{\sigma} b)$. In what follows, the subscript $i$ indexes cases. We begin with Case 1.

Case 1. For $p \leq v+\underline{\sigma} b$ and $p>\bar{\sigma} b$, the firm's demand is

$$
D(p, b)=1-\frac{p}{v}+\frac{b \mu_{\sigma}}{v} .
$$

Proof. Define $\hat{\theta}_{1}$ and $\tilde{\theta}_{1}$ as the points satisfying $\phi\left(\hat{\theta}_{1}\right)=\bar{\sigma}$ and $\phi\left(\tilde{\theta}_{1}\right)=\underline{\sigma}$, respectively, and recall that each point in $B \subset T$ has density $(\bar{\sigma}-\underline{\sigma})^{-1}$. Using Figure 1 (Panel A), one can see that consumers do not purchase the product with probability

$$
\frac{1}{(\bar{\sigma}-\underline{\sigma})}\left(\hat{\theta}_{1}(\bar{\sigma}-\underline{\sigma})+\frac{\left(\tilde{\theta}_{1}-\hat{\theta}_{1}\right)}{2}(\bar{\sigma}-\underline{\sigma})\right)=\frac{\hat{\theta}_{1}+\tilde{\theta}_{1}}{2} \text {. }
$$

Substituting

$$
\hat{\theta}_{1}=\frac{p-b \bar{\sigma}}{v} \quad \text { and } \quad \tilde{\theta}_{1}=\frac{p-b \underline{\sigma}}{v}
$$

into the preceding equation, we find

$$
\operatorname{Pr}\{\theta v+\sigma b \leq p\}=\frac{2 p-b(\underline{\sigma}+\bar{\sigma})}{2 v} .
$$

Recalling that $\sigma$ has expectation $\mu_{\sigma}=(\underline{\sigma}+\bar{\sigma}) / 2$, consumer demand can be written as

$$
D(p, b)=1-\frac{p}{v}+\frac{b \mu_{\sigma}}{v} .
$$

Next, we consider Case 2.

Case 2. For $p \leq v+\underline{\sigma} b$ and $p \leq \bar{\sigma} b$, the firm's demand is

$$
D_{2}(p, b)=1-\frac{(p-b \underline{\sigma})^{2}}{2 v(\bar{\sigma}-\underline{\sigma}) b}
$$

Proof. Define $\tilde{\theta}_{2}$ as the point satisfying $\phi\left(\tilde{\theta}_{2}\right)=\underline{\sigma}$. Using Panel B in Figure 1, consumers do not purchase with probability

$$
\frac{\tilde{\theta}_{2}(\phi(0)-\underline{\sigma})}{2(\bar{\sigma}-\underline{\sigma})}=\frac{(p-b \underline{\sigma})^{2}}{2 v(\bar{\sigma}-\underline{\sigma}) b} .
$$

Thus, demand is given by

$$
D_{2}(p, b)=1-\frac{(p-b \underline{\sigma})^{2}}{2 v(\bar{\sigma}-\underline{\sigma}) b}
$$


Next, we consider Case 3.

Case 3. For $p>v+\underline{\sigma} b$ and $p>\bar{\sigma} b$, the firm's demand is

$$
D_{3}(p, b)=\frac{(v-p+\bar{\sigma} b)^{2}}{2 v(\bar{\sigma}-\underline{\sigma}) b} .
$$

Proof. Define $\hat{\theta}_{3}$ as the point satisfying $\phi\left(\hat{\theta}_{3}\right)=\bar{\sigma}$. Using Panel C in Figure 1, consumers purchase with probability

$$
\frac{1}{2(\bar{\sigma}-\underline{\sigma})}\left[1-\phi\left(\hat{\theta}_{3}\right)\right][\bar{\sigma}-\phi(1)]=\frac{(v-p+\bar{\sigma} b)^{2}}{2 v(\bar{\sigma}-\underline{\sigma}) b} .
$$

Thus, demand is given by

$$
D_{3}(p, b)=\frac{(v-p+\bar{\sigma} b)^{2}}{2 v(\bar{\sigma}-\underline{\sigma}) b}>0
$$

Finally, we consider Case 4.

Case 4. For $p>v+\underline{\sigma} b$ and $p \leq \bar{\sigma} b$, the firm's demand is

$$
D_{4}(p, b)=\frac{v-2 p+2 \bar{\sigma} b}{2(\bar{\sigma}-\underline{\sigma}) b} .
$$

Proof. With the help of Panel D in Figure 1, one can easily show that consumers buy with probability

$$
\frac{1}{(\bar{\sigma}-\underline{\sigma})}\left(\bar{\sigma}-\phi(0)+\frac{\phi(0)-\phi(1)}{2}\right)=\frac{v-2 p+2 \bar{\sigma} b}{2(\bar{\sigma}-\underline{\sigma}) b}
$$

thus implying that demand is given by

$$
D_{4}(p, b)=\frac{v-2 p+2 \bar{\sigma} b}{2(\bar{\sigma}-\underline{\sigma}) b} .
$$

The next result derives the demand for prices $p \in(\underline{\sigma} b, v+\bar{\sigma} b)$.

Claim 1. Fix $b$ and suppose that $p \in(\underline{\sigma} b, v+\bar{\sigma} b)$. Then, demand is a piecewise-defined, differentiable function of $p$. If $\bar{\sigma} b \leq v+\underline{\sigma} b$,

$$
\hat{D}(p, b)= \begin{cases}D_{2}(p, b) & \text { if } p \leq \bar{\sigma} b \\ D_{1}(p, b) & \text { if } \bar{\sigma} b<p \leq v+\underline{\sigma} b \\ D_{3}(p, b) & \text { if } p>v+\underline{\sigma} b .\end{cases}
$$

If, instead, $\bar{\sigma} b>v+\underline{\sigma} b$, demand is given by

$$
\tilde{D}(p, b)= \begin{cases}D_{2}(p, b) & \text { if } p \leq v+\underline{\sigma} b \\ D_{4}(p, b) & \text { if } v+\underline{\sigma} b<p \leq \bar{\sigma} b \\ D_{3}(p, b) & \text { if } p>\bar{\sigma} b .\end{cases}
$$


Proof. Consider the case where $\bar{\sigma} b \leq v+\underline{\sigma} b$. Demand is continuous since the piecewise defined demand functions are continuous and

$$
\hat{D}_{2}(\bar{\sigma} b, b)=1-\frac{(\bar{\sigma}-\underline{\sigma}) b}{2 v}=\hat{D}_{1}(\bar{\sigma} b, b)
$$

as well as

$$
\hat{D}_{1}(v+\underline{\sigma} b, b)=\frac{(\bar{\sigma}-\underline{\sigma}) b}{2 v}=\hat{D}_{3}(v+\underline{\sigma} b, b)
$$

for all $b$. Differentiability follows by noting that there are no kinks in the demand curve:

$$
\left.\frac{\partial D_{2}(p, b)}{\partial p}\right|_{p=\bar{\sigma} b}=\left.\frac{\partial D_{3}(p, b)}{\partial p}\right|_{p=v+\underline{\sigma} b}=\frac{\partial D_{1}(p, b)}{\partial p}=-\frac{1}{v} .
$$

Now, consider the case where $\bar{\sigma} b>v+\underline{\sigma} b$. Then,

$$
\tilde{D}_{2}(v+\underline{\sigma} b, b)=1-\frac{v}{2(\bar{\sigma}-\underline{\sigma}) b}=\tilde{D}_{4}(v+\underline{\sigma} b, b)
$$

and

$$
\tilde{D}_{4}(\bar{\sigma} b, b)=\frac{v}{2(\bar{\sigma}-\underline{\sigma}) b}=\tilde{D}_{3}(\bar{\sigma} b, b)
$$

as well as

$$
\left.\frac{\partial D_{2}(p, b)}{\partial p}\right|_{p=v+\underline{\sigma} b}=\left.\frac{\partial D_{4}(p, b)}{\partial p}\right|_{p=\bar{\sigma} b}=\frac{\partial D_{4}(p, b)}{\partial p}=-\frac{1}{(\bar{\sigma}-\underline{\sigma}) b} .
$$

\section{A.2 Proof of Proposition 1}

(i) Differentiating the profit-maximizing product price, we have

$$
\frac{d p^{*}\left(\mu_{\sigma}\right)}{d \mu_{\sigma}}=\frac{4 v^{2} \gamma \mu_{\sigma}}{\left(4 v \gamma-\mu_{\sigma}^{2}\right)^{2}}>0
$$

(ii) Differentiating the level of advertising yields

$$
\frac{d b^{*}\left(\mu_{\sigma}\right)}{d \mu_{\sigma}}=\frac{v\left(\mu_{\sigma}^{2}+4 v \gamma\right)}{\left(4 v \gamma-\mu_{\sigma}^{2}\right)^{2}}>0 .
$$

(iii) Differentiating the profit function, we have

$$
\frac{d \pi^{*}\left(\mu_{\sigma}\right)}{d \mu_{\sigma}}=\frac{2 \gamma \mu_{\sigma} v^{2}}{\left(4 v \gamma-\mu_{\sigma}^{2}\right)^{2}}>0 \text {. }
$$

\section{A.3 Proof of Proposition 2}

The proof of (i) is immediate as $p^{*}$ does not depend on $\mu_{\sigma}$. (ii) Differentiating yields

$$
\frac{d D^{*}\left(\mu_{\sigma}\right)}{d \mu_{\sigma}}=\frac{v}{2\left(v-\mu_{\sigma}\right)^{2}}>0
$$

and

$$
\frac{d \pi^{*}\left(\mu_{\sigma}\right)}{d \mu_{\sigma}}=\frac{v^{2}}{4\left(v-\mu_{\sigma}\right)^{2}}>0
$$

